\title{
BIOCOMPATIBLE POLYMER EMBEDDED IN LIGHT-SENSITIVE MATERIALS: INVESTIGATION OF STRUCTURAL PROPERTIES
}

\author{
Gan Siew Mei ${ }^{1}$, Yuvaraj A.R. ${ }^{1}$, Siti Maznah bt Kabeb ${ }^{1}$, M.M. Yusoff ${ }^{1}$, V. G. \\ Chigrinov $^{2}$ and G. Hegde ${ }^{1 *}$ \\ ${ }^{1}$ Faculty of Industrial Sciences and Technology, Universiti Malaysia Pahang, \\ 26600, Pahang, Malaysia. \\ ${ }^{2}$ Department of Electronic and Computer Engineering, \\ Hong Kong University of Science and Technology, \\ Clear Water Bay, Hong Kong. \\ *Email: hegde@ump.edu.my \\ Phone: +60169844059; Fax: +6095492766
}

\begin{abstract}
Cost effective biocompatible polymer embedded in light-sensitive materials is reported for use in the photonics industry. Adhesion of photosensitive materials is improved by mixing them with highly viscous biocompatible substances. HEC (hydroxyethyl cellulose) is used as a biocompatible substance to study the photoalignment of light sensitive material SD-1. The mixture provides excellent results with good adhesion, better photoalignment quality and is highly suitable for plastic displays.
\end{abstract}

Keywords: HEC; order parameter; dichroic ratio; flexible display;

\section{INTRODUCTION}

Hydroxyethyl cellulose (HEC) is widely used for medical applications and is also used as a stabilizer and thickener in coatings. HEC is normally added as an additive in order to achieve the desired rheological effects, i.e., thixotropic behaviour during application of the coating [1-4]. The main thickener mechanisms of HEC in coatings are firstly through its solution properties, i.e., the rheology of a polymer in solution, and secondly through its adsorption properties, i.e., trains and loops on polymer particles and pigments particles, as well as limited bridging between various colloidal particles in the formulation [5]. Both the solution and adsorption behaviour of HEC are determined to a major extent by the molecular weight of the cellulosic backbone. Hence HEC is a wellknown biocompatible polymer. On the other hand, photoalignment has been proposed and studied for a long time. In fact, the subject of light and molecule interactions has been a fascinating subject of research for a long time and is still capturing the imagination of many people. Over the past twenty years, a lot of improvements and variations have been made for photoalignment [6, 7]. Many new applications, in addition to the alignment of LCDs, have been proposed and demonstrated. In particular, the application of photoalignment to photo-active compounds in optical signals processing and communications is currently a hot topic in photonics research. In spite of these potential advantages, there is still room for improvement and new materials are changing the way we are looking at things. A lot of work has been devoted to lightsensitive aligning materials (especially photoalignment) [8-11]. The alignment of liquid crystal (LC) molecules is an important research/development subject, because it is a key 
process in the fabrication of LC displays and devices [12]. Right from the beginning, the polyimide (PI) rubbing technique has been routinely used to align liquid crystals. It produces quite reliable alignment that is commonly acceptable for state-of-the-art devices. However, it cannot satisfy the strong demands of advanced technology. A principal drawback of this procedure is mechanical contact with the aligning substrate, which may destroy the alignment layer and surface electronics. Besides, it causes surface charging and dusting. The LC alignment produced by rubbing is not perfectly uniform, especially on the microscopic level. Because of this, alignment methods avoiding mechanical contact with the aligning substrates are being actively studied. Among the most promising candidates for future industry is photoalignment. This technique excels in terms of the high uniformity, wide range and smooth variation of the parameters of LC alignment. The preparation process of alignment layers made from traditional polyimide rubbing, however, requires high curing temperatures $\left(190-220^{\circ} \mathrm{C}\right)$ over a long time (about $30 \mathrm{~min}$ ) for completing the imidization (curing) process and therefore they are not appropriate for use in LCDs with flexible substrates which require a low curing temperature. Moreover, the shelf-storage time of these materials is about several months at $-10^{\circ} \mathrm{C}$ and their transportation is rather expensive since it requires special cooling conditions.

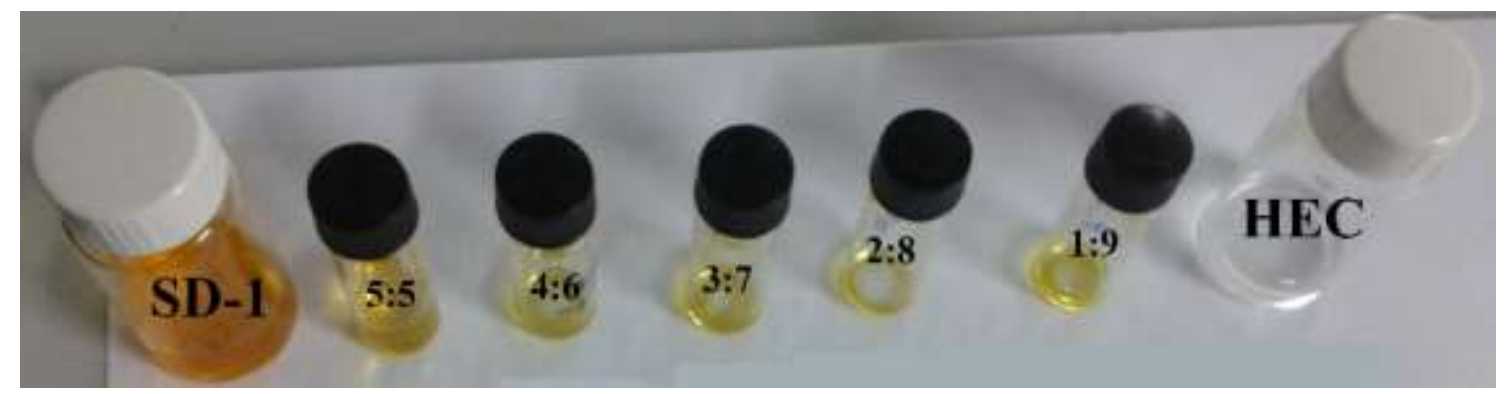

Figure 1. The series of solutions (SD-1: HEC) prepared by using different concentrations of SD-1 (1 wt $\%)$ and HEC (1 wt $\%)$.

The effect of LC photoalignment is a direct consequence of the appearance of the photo-induced optical anisotropy and dichroic absorption in thin amorphous films, formed by molecular units with anisotropic absorption properties [13]. Figure 1 demonstrates the basic concepts of photoalignment, where molecules are aligned in a particular direction when polarized UV light of a suitable wavelength is shone on them. The phenomenon of liquid crystal photoalignment became a subject of extensive research after the effect was discovered for the azobenzene units attached to a substrate [14] and dispersed in a polymer matrix [15-18]. Although a huge variety of photoaligning materials have been developed so far, nevertheless, azo dye materials remain among the best candidates for technological application. The azo dye SD-1 shows the best promise so far [6]. It possesses nice film-forming properties with HEC and high photosensitivity. As we show in this paper, the dye SD-1 is mixed with viscous material HEC to increase stability and adhesion. This study also suggests using these materials successfully for flexible displays, the so-called plastic displays. 


\section{EXPERIMENTAL}

\section{Sample Preparation}

To prepare alignment films, different ratios 1:9, 2:8, 3:7, 4:6, 5:5 of SD-1 and HEC were dissolved in water respectively with a fixed concentration. The solution was spincoated at $800 \mathrm{rpm}$ for 10 seconds and then at $1500 \mathrm{rpm}$ for 40 seconds onto substrates. Subsequently, the films were dried at $100{ }^{\circ} \mathrm{C}$ for 15 minutes.

\section{Experimental Procedure}

In the following stage, the films were irradiated with a linear polarized UV light from a mercury lamp. The polarized light was shone on the surface of the coated material with an intensity of $10 \mathrm{~mW} / \mathrm{cm}^{2}$ for an exposure time of 10 minutes. Parallel and perpendicular measurements were taken to find the order parameter and dichroic ratios for all thecompositions of SD-1:HEC. UV-Vis absorption spectra were recorded using a UV-Visible spectrophotometer obtained from Ocean Optics (HR2000+). The order parameter and dichroic ratios were investigated by illuminating with an OMNICURE S2000 UV source. The chemical structure of SD-1 and HEC is given below in Figure 2. HEC is commercially available, whereas SD-1 is obtained from Japan. Generally, HEC is a biocompatible polymer that is useful in several areas of research due to its particular properties like high viscosity, adhesiveness and thermal stability. SD-1 is a lightsensitive compound; it gives excellent photoalignment [6].

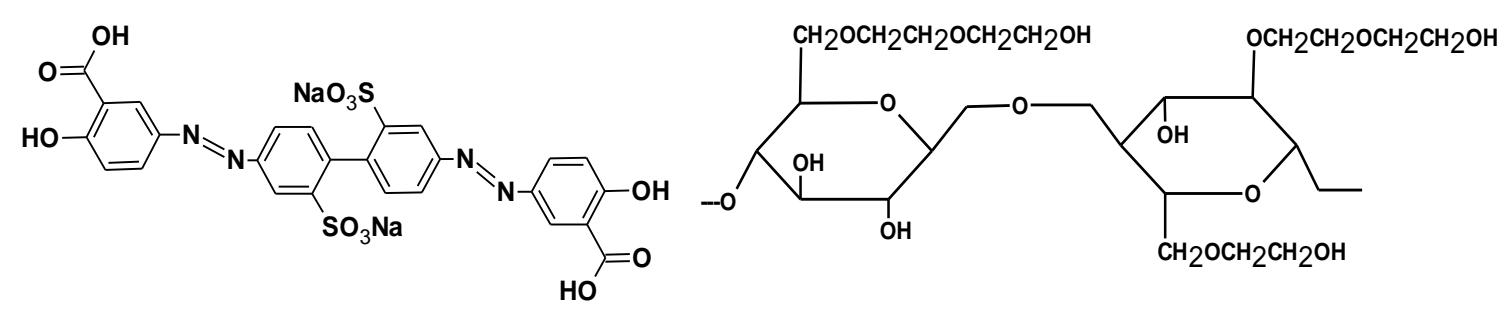

(a)

(b)

Figure 2. Chemical structure of SD-1 (a) and HEC (b).

The UV-Vis absorption spectra of HEC+SD-1 film showing the absorption maxima at $372 \mathrm{~nm}$ are shown in Figure 3. The absorption wavelength of all the ratios (SD-1:HEC) is about $\sim 372 \mathrm{~nm}$.

\section{RESULTS AND DISCUSSION}

Before irradiation, the absorption of the given composite alignment layer is independent of the polarization of light used in the measurements. After irradiation by linearly polarized UV light, the absorption of light shows polarization dependency. The absorption of light with its polarization direction parallel $\left(\mathrm{D}_{\|}\right)$to the UV light polarization direction decreases, whereas the absorption of light with its polarization direction perpendicular $\left(D_{\perp}\right)$ to the UV light polarization direction increases. 


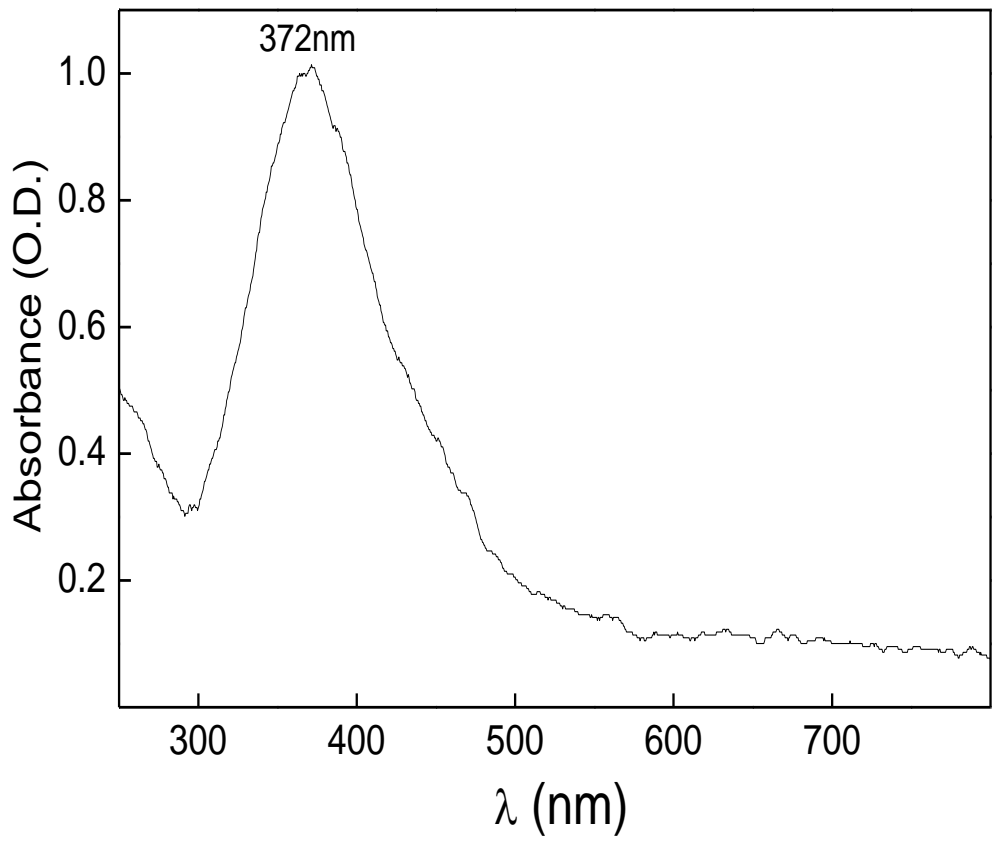

Figure 3. UV-Vis absorption spectra of HEC+SD-1 film showing that the absorption of 1:1 mixture of SD-1:HEC is about $\sim 372 \mathrm{~nm}$ maxima at $372 \mathrm{~nm}$ absorption wavelength.

The dichroic ratio (DR) and order parameter (S) of the azo dye chromophores were calculated using Eq. (1) and (2), where $D_{\|}$and $D_{\perp}$ are the absorption of parallel and orthogonal polarized light with respect to the polarization of the actinic UV light, respectively. The order parameter $\mathrm{S}$ can be expressed in Eq. (1).

$$
\mathrm{S}=\left(\mathrm{D}_{\|}-\mathrm{D}_{\perp}\right) /\left(\mathrm{D}_{\|}+2 \mathrm{D}_{\perp}\right)
$$

The dichroic ratio is defined as the ratio of perpendicular absorption to the parallel absorption. It is given in Eq. (2).

$$
\mathrm{DR}=\mathrm{D}_{\perp} / \mathrm{D}_{\|}
$$

where $D_{\|}$and $D_{\perp}$ are the absorption (optical density) of parallel and orthogonal polarized light to the polarization of the activated UV light [15].

Before mixing any biocompatible mixture, experiments were carried out only on original SD-1 molecules, and their dichroic ratio and order parameter are given in Figure 4, which shows the good values of the order parameter and dichroic ratio for these compounds. Later, experiments were done by mixing HEC with SD-1. The investigations on the optical activity of the different ratios of SD-1 and HEC are presented in Figure 5 and Figure 6 . The mixtures exhibit excellent photoalignment effects. According to the theory, photoalignment is enhanced with a higher order parameter and higher dichroic ratio. Here HEC is used to increase the adhesion on the given substrates. 


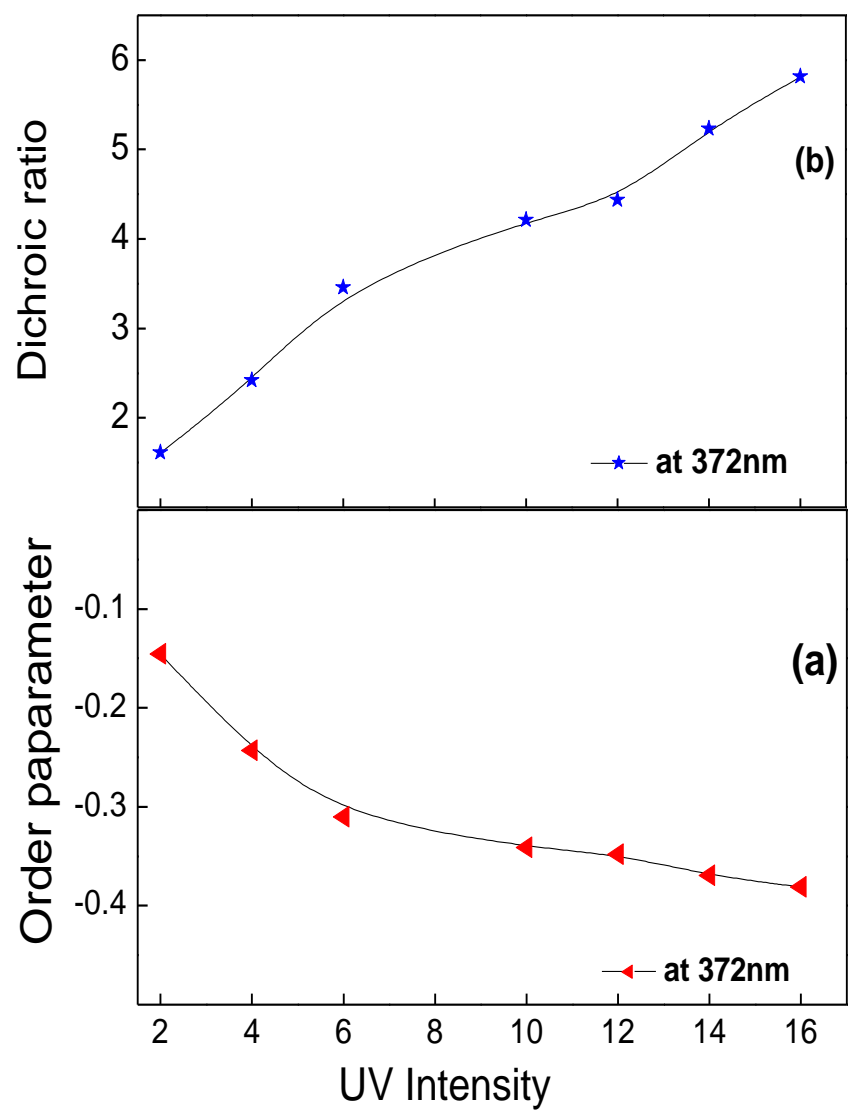

Figure 4. Variation in the order parameter (a) and dichroic ratio (b) for compound SD-1 with respect to intensity. A linear polarized UV light passing through a $365 \mathrm{~nm}$ filter is shone for the sample.

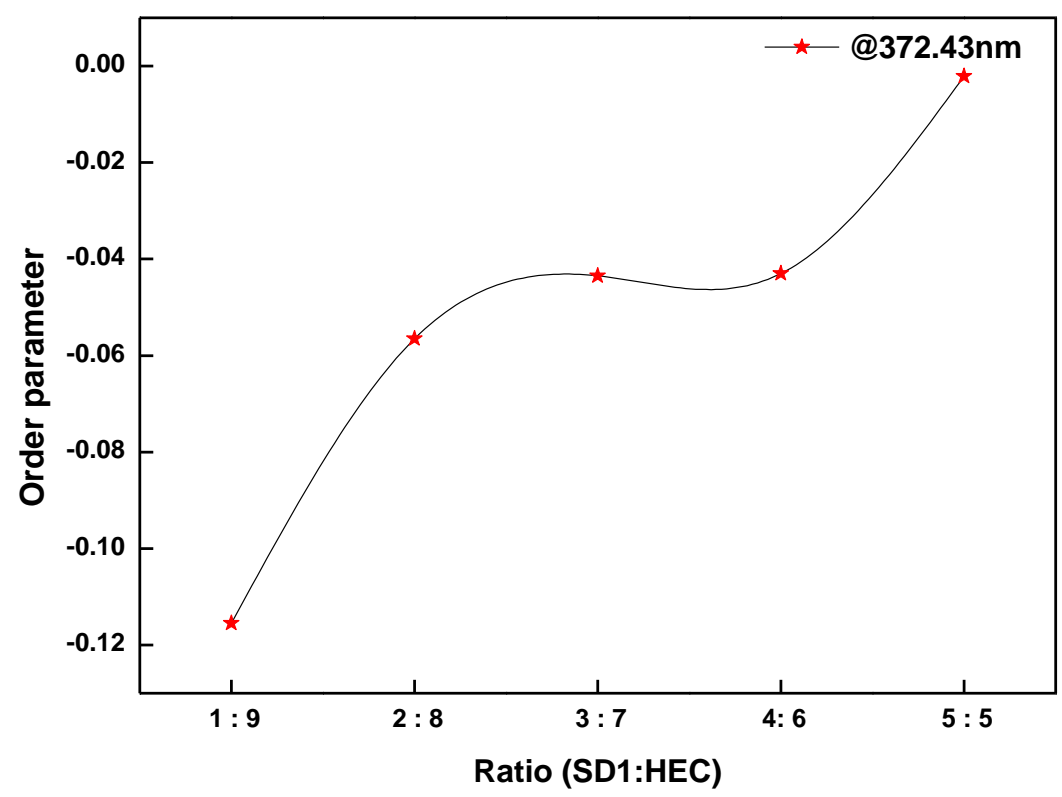

Figure 5. The order parameter with respect to the different ratios of SD-1:HEC is determined with fixed wavelength and fixed exposure time. 


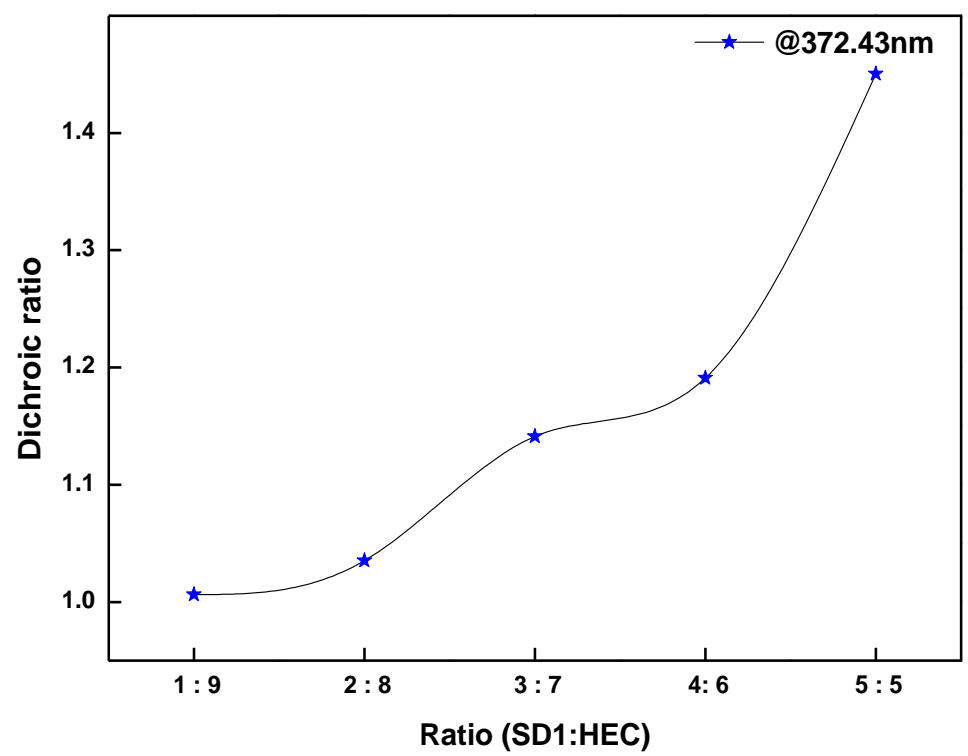

Figure 6. The dichroic ratio with respect to the different ratios of SD-1:HEC is determined with fixed wavelength and fixed exposure time.

Along with these experiments, it is proved that the 7:3 (SD-1:HEC) ratio is the best combination for coating, since it exhibits excellent photoalignment results, as shown in Figure 7. Moreover, the 7:3 (SD-1:HEC) combination gives good adhesion with quartz, glass and plastic substrates. Hence one can make more stable flexible devices by using the 7:3 (SD-1:HEC) mixture with the principle of photoalignment. In Figure 7 , one can see the higher dichroic ratio and order parameter with the gradually increasing concentration of light-sensitive material SD-1.

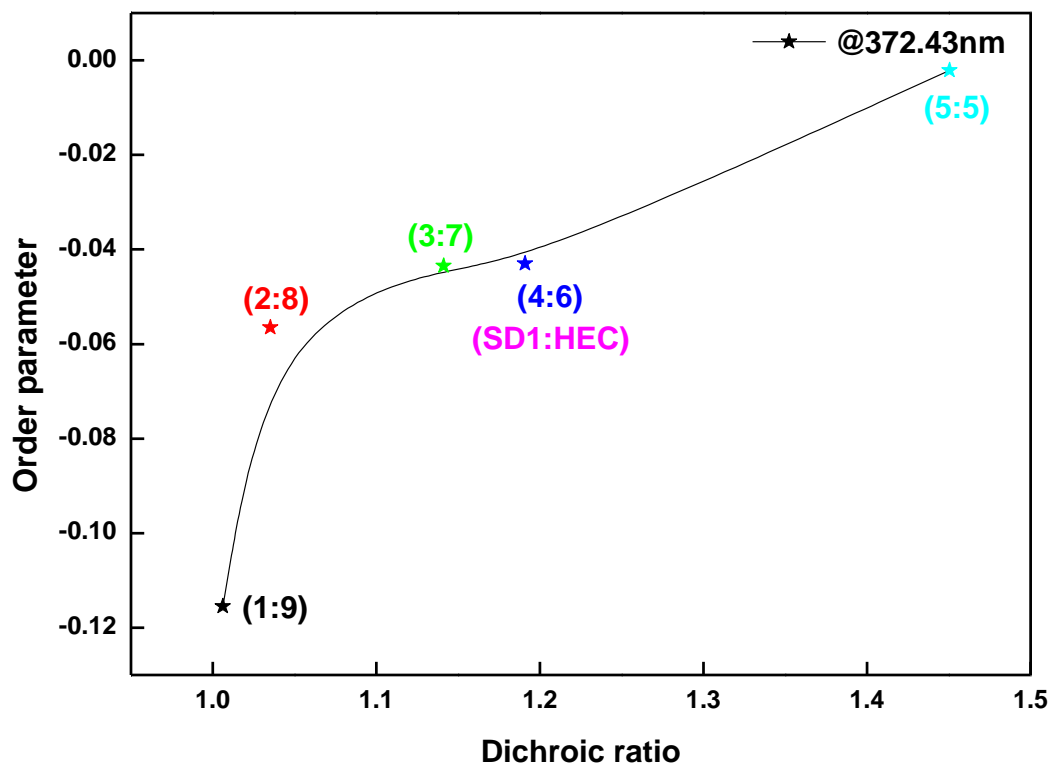

Figure 7. The order parameter and dichroic ratio for every composition of (SD-1:HEC) with fixed wavelength and fixed exposure time. 
So the presented data provides direct evidence of the importance of biocomposite mixtures and their inherent characteristics. Using these results, a prototype is constructed with the procedure described in this article. Figure 8 shows the photograph of a bright state containing alignment layer made from the composite mixture 7:3 (SD$1:$ HEC) and filled with different modes of liquid crystals used especially in the liquid crystal display industry. Cells were inserted in between two crossed polarizers. The dark state corresponds to its position between crossed polarizers with the cell optic axis oriented along one of the polarizers, whereas the bright state will appear after rotating the cell by $45^{\circ}$. The liquid crystal alignment in the cells is perpendicular and it shows excellent contrast.
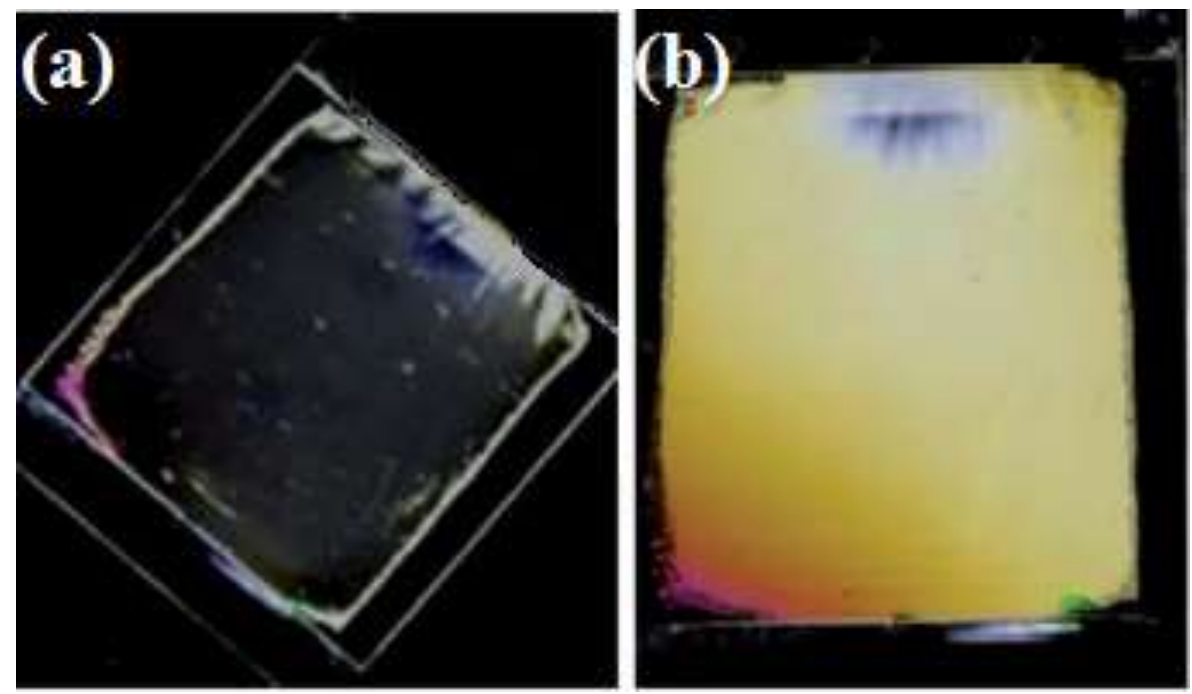

Figure 8. Images of flexible cell made using 7:3 SD-1 and HEC. (a) The dark state corresponds to its position between crossed polarizers with the cell optic axis oriented along one of the polarizers. (b) The bright state appears after rotating the cell by $45^{\circ}$.

Although we don't know the exact reason for this excellent alignment behaviour, we speculate the following. In the light of photoalignment effects, it is always difficult to control adhesion and most of the time this adhesion will prevent uniform coating. When we mix biocompatible HEC having a high viscosity with less viscous lightsensitive materials, a good mixing will take place between the two materials. When you coat this controlled viscous mixture, one can coat any thickness in any condition in a uniform manner. Biocompatible materials might help the light-sensitive molecules to sit in proper channels when linear polarized light of a suitable wavelength is shone on them. This is again dependent on concentration, since not all concentrations may be suitable to get useful photoalignment effects due to the high viscosity behaviour of HEC. Controlling the viscosity is dominant factor when using biocomposite mixture. So the presented method is a cost-effective way of aligning display materials, thereby allowing the fabrication of mobile screens, television screens and laptop screens without great cost.

\section{CONCLUSIONS}

In summary, biocompatible material incorporated in dye material plays a crucial role in aligning the surface. Controlling the viscosity seems to be the dominant factor in this 
mixture since a higher viscosity destroys the alignment completely, whereas a lower viscosity is unable to coat the substrates. Adhesion is drastically improved using this mixture due to the user-friendly nature of the viscosity control. The thermal stability of the photoalignment of the given composite mixtures could be further improved by using different photoreactive materials with the HEC. This study shows that the mixture of HEC and photoreactive composite will be helpful in the production of flexible displays for future generations of the electronics industry.

\section{ACKNOWLEDGEMENTS}

This work was supported by University Malaysia Pahang RDU grant number RDU 131403.

\section{REFERENCES}

[1] Arfin N, Bohidar H. Concentration selective hydration and phase states of hydroxyethyl cellulose (HEC) in aqueous solutions. International journal of biological macromolecules. 2012;50:759-67.

[2] Ab Latif N, M. Rus AZ. Vibration transmissibility study of high density solid waste biopolymer foam. Journal of Mechanical Engineering and Sciences. 2014;6:772-81.

[3] Hardinnawirda K, SitiRabiatull Aisha I. Effect of rice husks as filler in polymer matrix composites. Journal of Mechanical Engineering and Sciences. 2012;2:181-6.

[4] M. Khairul Zaimy AG, Zafiah A, Rus M, Ab Latif N, Nurulsaidatulsyida S. Mechanical and thermal properties of waste bio-polymer compound by hot compression molding technique. Journal of Mechanical Engineering and Sciences. 2013;5:582-91.

[5] Abdel-Halim E. Preparation and characterization of poly (acrylic acid)hydroxyethyl cellulose graft copolymer. Carbohydrate polymers. 2012;90:930-6.

[6] Chigrinov VG, Kozenkov VM, Kwok H-S. Photoalignment of liquid crystalline materials: physics and applications: John Wiley \& Sons; 2008.

[7] Hegde G, Alla RA, Matharu A, Komitov L. Azo containing thiophene based prop-2-enoates for photoalignment of a nematic liquid crystal. Journal of Materials Chemistry C. 2013;1:3600-5.

[8] Yaroshchuk O, Reznikov Y. Photoalignment of liquid crystals: basics and current trends. Journal of Materials Chemistry. 2012;22:286-300.

[9] Goodby JW. The nanoscale engineering of nematic liquid crystals for displays. Liquid Crystals. 2011;38:1363-87.

[10] Zhang L, Sun X, Liu Y, Peng Z, Xuan L. Photoalignment of liquid crystals by a covalently attached self-assembled ultrathin film. Liquid Crystals. 2012;39:98391.

[11] Sato S IH, Mizunuma T, Nagai K, Matsumoto $H$, Matsumoto $\mathrm{S}$. Hexafluoroisopropylidene group on photoalignments of liquid crystal molecule. Journal of Photopolymer Science and Technology. 2011;24:617-23.

[12] Akiyama H, Kawara T, Takada H, Takatsu H, Chigrinov V, Prudnikova E, et al. Synthesis and properties of azo dye aligning layers for liquid crystal cells. Liquid Crystals. 2002;29:1321-7. 
[13] Li X, Kozenkov VM, Yeung FSY, Xu P, Chigrinov VG, Kwok HS. Liquidcrystal photoalignment by super thin azo dye layer. Japanese journal of applied physics. 2006;45:203.

[14] Pozhidaev E, Chigrinov V, Hegde G, Xu P. Multistable electro-optical modes in ferroelectric liquid crystals. Journal of the Society for Information Display. 2009;17:53-9.

[15] Chigrinov VG, Kozenkov VM, Kwok HS. New developments in photo-aligning and photo-patterning technologies. In: Vicari L E, editor. Optical Applications of Liquid Crystals: Taylor \& Francis; 2003.

[16] Adebisi AA, Maleque MA, Rahman MM. Metal matrix composite brake rotor: Historical development and product life cycle analysis. International Journal of Automotive and Mechanical Engineering. 2011;4:471-80.

[17] Mat Hassan NN, M. Rus AZ. Acoustic performance of green polymer foam from renewable resources after UV exposure. International Journal of Automotive and Mechanical Engineering. 2014;9:1639-48.

[18] Mohamed WANW, Atan R. Polymer electrolyte membrane fuel cell. International Journal of Automotive and Mechanical Engineering. 2012;5:64859. 